\title{
An In-Depth Study of TCA cycles, 0PA1, S6K1, ATPase, TLR4, MHC-class-I, GCs, and IFNs Biosynthesis, and Their Roles of Deficiency in Diabetes, Asthma, Cancer, etc. and the NAD Roles in their Activities
}

\author{
Ashraf Marzouk El Tantawi* \\ Biomedical Molecular Studies, Egypt
}

\begin{abstract}
Background: Plummer Vinson syndrome is a rare cause of dysphagia in children. It associates an oesophageal organic stenosis to a sideropenic anemia.

Case Reports: Two Tunisian boys aged respectively 14 and 9 years-old were admitted to investigate dysphagia for solid foods. The adolescent patient was a black and he suffers from food blocking since 2 years ago. The assessment revealed a sideropenic anemia due to intestinal giardiasis. While the second patient was followed for a chronic anemia since he was 3 years old. For both of them, the swallow barium exploration showed an annular stenosis of the cervical esophagus mimicking "a thread knotted on a round tube" related to a circular web identified by fibroscopy. The iron supplementation and dilatation by the esophagoscope allowed the resolution of the clinical signs.
\end{abstract}

Conclusion: Plummer Vinson syndrome exists in paediatric population. It should be evoked in any children presenting with upper dysphagia for solid foods in order to initiate iron supplementation and to watch for cancerous degeneration by regular followup.

KEYWORDS: Plummer vinson; Dysphagia; Oesophageal stenosis; Anemia; Children

\section{PORPOSE OF THIS STUDY}

The roles of S6K1 are regulating ATPase, abd GTPase synthesis, and consequently regulate endocytic soluble MHC class II synthesis and SIRP 1 which promote TLR4 synthesis. Diabetes reflect deficiency in pyrimidines synthesis consequently deficiency in Estrogen with increasing in androgen synthesis that after period of time will increase consuming purines (A\&G) in ATPase synthesis that led to decreasing in anabolic processes and proliferations too.

OPA1-synthetase enzymes are so important for producing amino-acyl-CoA-synthetase (gamma-subunits) and signals, where gamma-subunits are the basics for regulating glucocorticoids synthesis, and Interferon's isoforms productions, for reactivating macrophages, through MHC class-I synthesis which migrate to nucleated cells for endocytic soluble MHC class II synthesis which promote both SIRP $\alpha 1$ and TLR4 synthesis upon phospholipase effects on Beta-subunit "fatty-acyl-CoA-synthase subunits". S6K1 consider as protected basic subunits for Interferons (IFNs) isoforms synthesis regulated by pyrimidine-kinases. Asthma is characterized by Prediabetes and diabetes, that reasons of the occurrence of the two diseases (Asthma \& diabetes) are same which is deficiency in Ser amino acids and deficiency in pyrimidine synthesis which regulated by synthetase enzymes. Estrogen formed from Ser/ThrFOX signaling pathways for glucocorticoids synthesis (and for IFNs synthesis) Upon OPA1 enzymes effects on estrogen.
Quick Response Code:

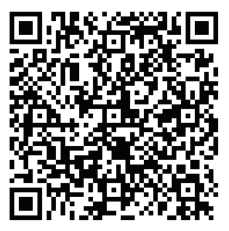

Address for correspondence: Ashraf Marzouk El Tantawi, Biomedical Molecular Studies, Toronto Canada, and Cairo Egypt

Received: September 22, $2021 \quad$ Published: October 11, 2021

How to cite this article: Ashraf M El T. An In-Depth Study of TCA cycles, OPA1, S6K1, ATPase, TLR4, MHC-class-I, GCs, and IFNs Biosynthesis, and Their Roles of Deficiency in Diabetes, Asthma, Cancer, etc. and the NAD Roles in their Activities. 2021- 3(5) OAJBS.ID.000331. DOI: 10.38125/OAJBS.000331 


\section{INTRODUCTION}

The purines and pyrimidines synthesis in vivo are the main basic processes for all of proper active subunits and active genes biosynthesis and for all cellular biological processes where any delays in their production or deficiency in some of their purines or pyrimidine will lead to symptoms of diseases, whether dangerous or benign. Mitochondrial TCA cycles are a regulated metabolite related and depending on FOX genes functions and on ATPase with cox activities on carbohydrate, on lipid, and on proteins pronutrients-mTOR molecules for producing first long fatty acids chains and lipo-polysaccharides (LPS molecules), where, as FOX genes produce the four GPS of kinases (PSTCk, PSTTK, PSTGk and PSTA-kinases), as the mitochondrial enzymes (synthetase, synthase and phospholipase) will start their oxidative processes for modifying those four kinases GPS for producing only three fatty acyl-CoA isoforms.

The full releasing of PSTGk and PSTAk GPS depending on FOX functions and on ATPase with COX enzymes for S6K1 peptides synthesis (which are formed from purines mainly adenosine nucleotides for ATPase production and repair) which will be directed to ribosome granules within cells for further endocytic modification efforts by OPA1 enzymes for firstly pyrimidine synthesis then for producing gamma-subunits upon Gamma-oxidations by synthetase enzymes, where, I consider that the releasing of PSTGk and PSTAk are depending on the TCA cycles for producing purines kinases (PS/ T-Adenosine kinase and PS/T-Guanosine kinases) or acyl-CoAs rich of purines, (After mitochondrial OPA1 modification effects ), where
TCA produce purines which will be used with pyrimidine (which produced from synthetase effects on LPS and on fatty long kinases proteins )for hydrophobic acids synthesis. normally the releasing of PS/T-Thymine -Kinase and PS/T-Cytosine -kinase chains (mTORC1) are the main for hydrophobic amino acids synthesis then modified by synthetase enzymes with purines which produced from TCA cycles for the necessary hydrophobic acids synthesis for rebuilding the first gamma-subunits which promote the synthesis of Betasubunit and alpha subunits Upton synthase and phospholipase respectively.

S6K1 synthesis and functions controls tubulin acetylation (Due to S6K1 is main regulator active peptides for ribosomal repairs and for ATPase productions), hence S6K1 active subunits are contributing to the Autophagic flux induced by different stress conditions and in different cells. mTORC1 is one of the two substrates (The basis for regulating S6K1 peptides productions) of regulating and stimulating ribosomal S6protein-kinase-1 (S6K1) synthesis through Fox Serine/Threonine signaling activated pathways in response to nutrients and growth factors where the second substrate needed for S6K1 production is the PS/T-Thymine Kinases. The availabilities of proline (Pro), Arg, Ser, Thr, Leu, and Tyr amino acids in S6K proper peptides and in Thymine kinases with cytosine kinases will enhance the proper activity of S6K1 and hence the proper functions of ATPase which regulate anti-inflammatory activities started by ATPase synthesis and GTPase which can prevent cancer, coagulation, prevent platelets aggregation, prevent bones weaknesses and erosion of the vertebrae, and prevent diseases symptoms (Figure 1).

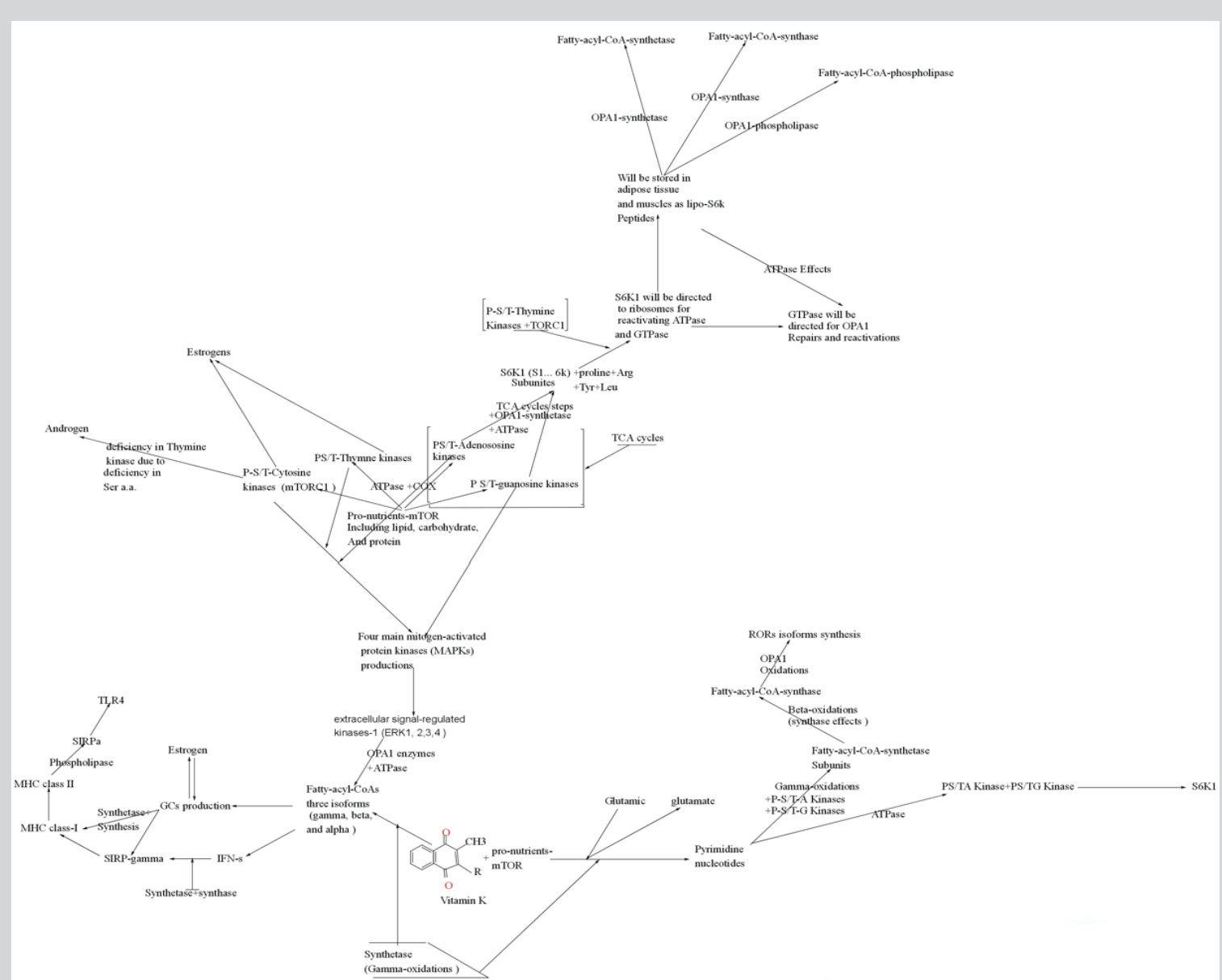

Figure 1: S6K1 synthesis regulated by mTORC1 + P-S/T-Thymine kinases Productions bonded with purines which produced from TCA Cycles. 
$\mathrm{NA}+, \mathrm{K}+$-ATPase membrane bonding enzymes (MBE) adopt hypertension. where, the N'A+, K+ ATPase (MBE) functions are stabilizing cells membranes signals activities and adopt the negative charges signals transmission that adopt and stabilizing brain activities. Estrogen biosynthesis depending on Ser/Thr mTOR Fox signaling pathways for glucocorticoids synthesis (the substrate of GCs synthesis) Upon OPA1 enzymes effects on Estrogen, but the feedback of GCs to produce estrogen will be upon ATPase and synthetase regulations on GCs isoforms. S6K1 consider as protected basic subunits for Interferons (IFNs) isoforms synthesis regulated by pyrimidine-kinases. Asthma is characterized by Pre-diabetes and diabetes, that reasons of occurrence the two diseases are same. Where, positively Asthma is characterized by diabetes symptoms May in the early days of Asthma cannot be detected but after early days of diabetes can be detected. MHC Class-I is fatty-acyl-CoA gamma-subunits (IFN-gamma) for modifying their fatty acyl-CoAbeta (IFN-beta) synthesis to be sit and lie on nucleated cells as MHC class-1 for endocytic MHC class-II production which will promote SIRP $\alpha$ synthesis for TLR4 productions. Signal regulatory proteinalpha, SIRP (SIRP-alpha) is the basic for producing IFN-beta then IFN-alpha which activate TLR4 for Plasma membrane synthesis where through feedback will produce firstly MHC class II (IFN-beta upon synthase beta-oxidation) followed by gamma-oxidation for producing MHC Class-I.

SIRP-gamma (IFN-gamma) productions from myeloid is the first SIRP molecular structure that produced that will promote MHC-I under the regulations of IFN-gamma and beta that are necessary for acting fast on inflammations and infection-ed cells for analyzing their contents then bind to resulted peptides for modifying its own sensor subunits for promoting the re-producing of MHC class-I and thenSIRP-2-Beta (IFN-beta) upon regulations effects of OPA1 synthase enzyme which will directed to nucleated cells membranes to sit and lie on as MHC class-I which will promote the endocytic soluble MHC class II synthesis .The role of functions of the OPA1 oxidations cycles in producing S6K1 for ATPase for producing and for functioning the fattyacyl-CoAs for producing NADH and then ATPase. Where, decreasing or deficiency in S6K1 production will reflect the decreasing in the ATPase which also reflect the decreasing in mitochondrial OPA1-oxidations and decreasing in NAD and FAD that reflect the beginning of many diseases as asthma, diabetes, cancers, etc.

\section{MATERIALS}

- $\quad$ FOX genes,

- $\quad$ S6K1, ATPase and GTPase

- $\quad$ OPA1 enzymes

- $\quad$ MTORC1, PS/T-Thymine-K, PS/T-Adenosine-k, PS/TGuanosine-kinases

- Fatty-acyl-CoA-synthetase,

- Fatty-acyl-CoA-synthase,

- Fatty-acyl-CoA-phospholipase

- $\quad$ N'A, K, ATPase

- Renal proximal tubule epithelia

- Beta-cells and beta chains

- $\quad$ CD8 T cells
- IFN-gamma, IFN-beta, and IFN-alpha

- $\quad$ Diabetes and asthma studies

- $\quad$ MHC Class-I, MHC class II

- $\quad$ Signal proteins

- $\quad$ Tyrosine metabolic pathways

- $\quad$ Arg metabolism and proline synthesis

- $\quad$ Mast cells

- $\quad$ Nicotinamide adenine dinucleotide, oxidized form (NAD+)

- $\quad$ T-cells

- B-cells

- $\quad$ TGF-gamma, beta, alpha

- $\quad$ TLR4

- $\quad$ Myeloid cells

- $\quad$ SIRP-gamma, SIRP-beta, SIRP- $\alpha$

- $\quad$ Phosphatidic acid (PA)

\section{METHODS AND RESULTS}

TCA cycles improves antibody, cytokines, and acetyl CoA production [1]. Mitochondrial TCA cycles are a regulated metabolite, which considered as apart from FOX genes activities and depending on FOX functions, on ATPase, and on OPA1 enzymes activities on carbohydrate, on lipid, and on proteins nutrient molecules. where, as FOX genes produce the four GPS of kinases (PS/T-Cytosine kinases, PS/T-Thymine Kinases, PS/T-Guanosine kinases and PS/T-Adenosine kinases) as mitochondrial enzymes will start their oxidative effect for modifying those four kinases GPS for producing only three fatty acyl-CoA isoforms (Figure 2). The full releasing of PS/TGk and PS/TAk GPS depending on FOX functions and on ATPase with COX enzymes I considered their biosynthesis pathways regulated by OPA1 enzymes functions as necessary part of TCA cycles for producing purines kinases or acyl-CoAs full of purines, where, TCA cycle concluded the release of PS/T-T-Kinase and PSTC-kinase (mTORC1), and the synthesis of fatty-acyl-CoAsynthetase subunits which are depending on synthetase enzymes effects for pyrimidines synthesis (cytosine and Thymine's) for hydrophobic amino acids [2].

The FOX genes pathways functions and ATPase with COX oxidative processes are necessary for producing the necessary four kind of kinases GPS which then followed by mitochondrial enzymes effects for producing three fatty-acyl-CoA- isoforms started by OPA1 synthetase enzymes effects - gamma-oxidation) for producing fatty-acyl-CoA-synthetase (gamma-subunits), that their proper activities are related to and depending on the presence of purines with pyrimidine (which produced from synthetase functions) for hydrophobic amino acids synthesis where some of those necessary amino acids are: Alanine amino acids (GCC,GCA,GCG) (which necessary for alanine-amino transferase) and necessary for migrating molecules and the synthesis of mTORC1 (P-S/TCytosine kinases), Arg (AGA,AGG) (which is necessary for ATPase and GTPase synthesis and reactivities), Ser (AGT,AGC)(which is necessary for both cytosine kinases and thymine kinases synthesis), Thr, leu, (which is so necessary for estrin synthesis and brain activities) and Tyr amino acids synthesis (which considered 
as imp hydrophobic amino acids and imp for regulating most of active subunits and genes), where the purifications and synthesis of purines nucleotides (guanine and adenosine) from TCA cycle is a part of FOX pathways which promote the production of the two kinases adenosine kinases and guanosine kinases for the next steps of the mitochondrial OPA1 synthetase effects (gamma-oxidations) for necessary pyrimidine synthesis for hydrophobic amino acids synthesis. So, I consider that synthetase enzymes can adopt and regulate TCA cycles through functioning purines for purines kinases PS/TGk and PS/TA kinases then for hydrophobic acids synthesis in the necessary protein kinases subunits (eg: S6K1, and other cytokines productions upon the effects of synthetase enzymes) and in the fatty-acyl-CoA isoforms (Figure 3).
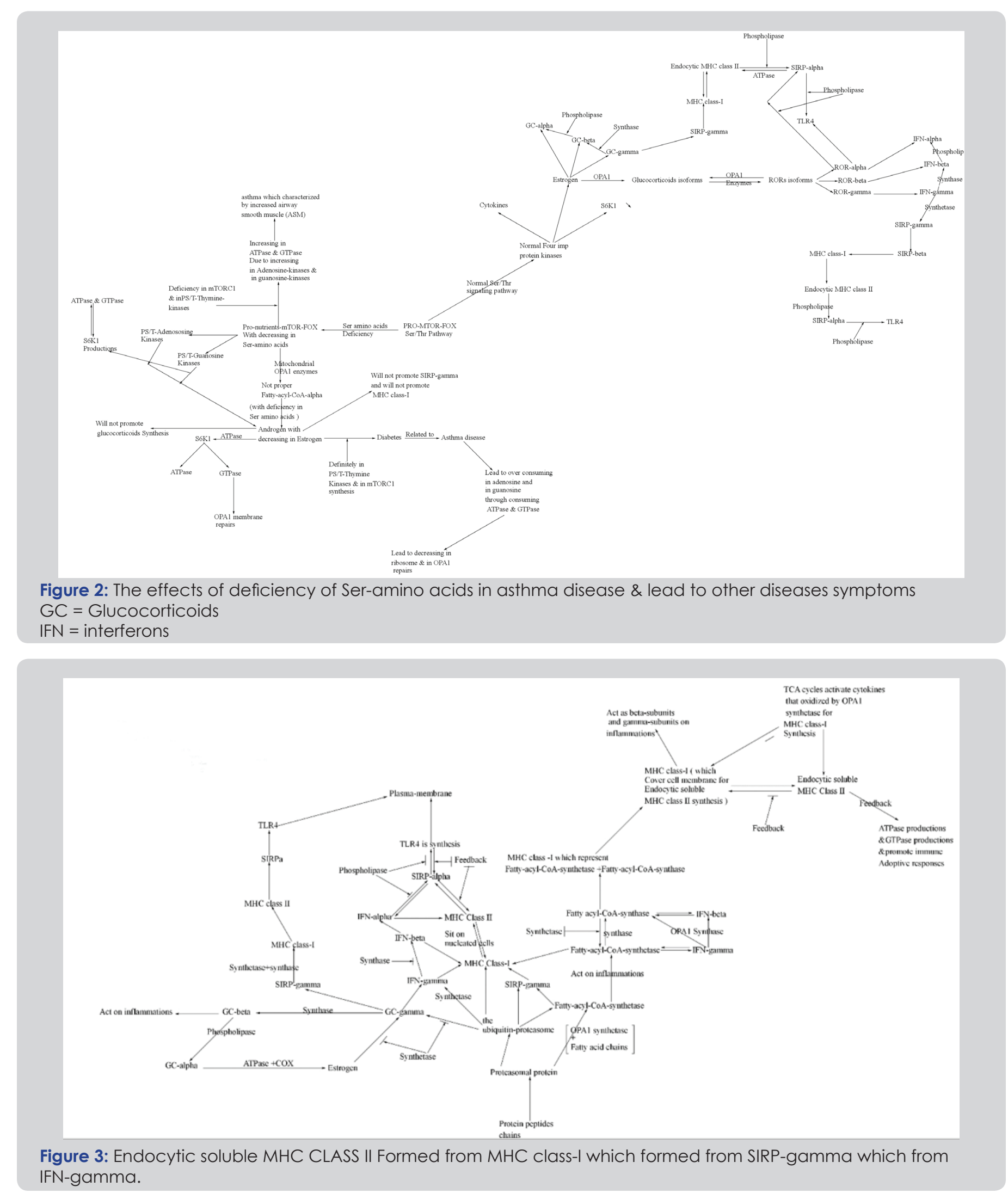
TCA cycle metabolites are centrally important determinants of macrophage metabolic re-programming [3]. It has been reported that Metabolism in immune cells is no longer thought of as merely a process for adenosine triphosphate (ATP) production [4], but I reported that ATPase productions are so necessary for all cells biosynthesis and metabolism specifically immune cells for activating their ATPase productions which necessary for creating their necessary active signals transmission for stimulating other cellular biosynthesis and for GTPase productions which necessary for mitochondrial OPA1 repairs. ATPase productions started by the formation of S6K1 peptides which is regulated by P-S/T-Adenosinekinases and by P-S/T-Guanosine-kinases subunits, and by mTORC1 together productions and can be stored in ribosome as S6K1 which upon phosphorylation will produce ATPase and GTPase that will be directed for repairing OPA1 inner membrane that will re-activate proper gamma-oxidation for producing proper necessary gammasubunits or fatty-acyl-CoA-gamma which necessary for Betasubunits productions upon the synthase effects(beta-oxidations).

Where, the death of living cells started by broken ribosomes or by analyzing RNAs without repairs and through re-producing ATPase or GTPase from their main S6K1, that will lead mutations or stopping producing acyl-CoA isoforms that can reflect Brocken pathways including TCA cycles and increasing FOX activities that will stop releasing PS/TGk and PS/TAk cytokines (which produced upon ATPase and COX enzyme oxidative processes) that will lead to cells death or pathogenic symptoms. Also, notice that Tubulin acetylation which regulated by ATPase plays important role in cellular activities including cell polarities that tubulin acetylation forms ATPase and GTPase and depending on ATPase with GTPase productions that reflect healthy status of living cells and reflect the production of S6K1 by tubulin acetylation.

Also, S6K1 synthesis and functions can controls tubulin acetylation (due to S6K1 is main regulator active peptides for ribosomal repairs and ATPase productions), hence, S6K1 active subunits can contributing to the Autophagic flux induced by different stress conditions and in different cells. During tubulin acetylation, S6K1 contributes to the flux of autophagy induced by different stress conditions and in different cells, where this effect appears to be independent of the kinase activity of S6K1 [5]. S6K1 biosynthesis can reflect creations of signals effective transmission which can stimulate and promote gamma-oxidation upon the effects of OPA1 synthetase for producing amino-acyl-CoAsynthetase (gamma-subunits) with enough energy that simplified in the created active signals which can be used for migrating the produced subunits and stimulating the beta-oxidations (upon synthase effects on gamma-subunits).

The effects of OPA1-synthetase (OPA1Stase) will be the results of pyrimidine synthesis including cytosine and active signals transmission which will be used for mTORC1 (PSTCk) subunits productions which will be necessary for regulating the biosynthesis of the S6K1 peptides, thenS6ks will be directed for endocytic biosynthesis for repairing and re-activating rRNAs and for ATPase and GTPase productions for mitochondrial OPA1 membrane repairs and for the production of acyl-CoA isoforms upon OPA1 enzymes effects and are so necessary for controls tubulin acetylation and for regulating axon growth through beta-oxidations (for beta-subunits productions) followed by alpha-oxidations which produce alpha subunits necessary for axon growth and autophagy biosynthesis.

Also, the conversion of ketoglutarate to glutamate is imp for the acetylated tubulin-NKA complex and is so imp for proline synthesis which regulated by TCA cycles. After, ATPase Effects started on pro-nutrients-mTOR and started to produce S1. 6-Kinases. Which can be considered as a basis of variety of cytokines with different compositions. The mammalian target of rapamycin (mTOR) positively regulates axon growth [6], where mTORC1 is the main of regulating and stimulating ribosomal S6-protein-kinase-1 (S6K1) synthesis through Fox Serine/Threonine signaling activated pathways. And the maintaining of glucose supply for purines synthesis necessary for S6K1 protein synthesis is necessary for central nervous system through for the formation of the three acyl-CoAs (isoforms) for fatty acid synthesis [7]. The maintaining of glucose supply (purines supply) is necessary for reactivating the long fatty acids chains productions upon ATPase effects and COX effects, and for S6K1 synthesis (as I mentioned previously) which necessary for controlling platelet activation and aggregate formation [8].

Maintaining glucose supply (purines supply) are necessary for ATPase reactivation through regulating S6K re-production (with proper composition from hydrophobic amino acids example of proline, ser, Arg, Leu and tyrosine) which is necessary for reactivating GTPase synthesis. The availabilities of proline (Pro), Arg, Gly, Asp (GAC), Ser, Thr, Leu, and Tyr amino acids are so necessary for S6K proper activities where enhance the proper functions of ATPase and OPA1 oxidative processes (Gamma, beta, then Alpha) which can analyze tumors (upon synthetase effects), coagulation, prevent platelets aggregation, prevent bones weaknesses and erosion of the vertebrae, and prevent diseases symptoms. Also, Asp (GAC) is necessary for deliver its purines $A \& G$ by cytosine to endocytic ribosomes (where, Cytosine imp for tRNAs productions and functions). The proper ATPase composition is necessary for amphetamine-based anorectic which necessary to maintain neuronal excitability which can rapidly converted into amphetamine in vivo. $\mathrm{Na}+, \mathrm{K}+$-ATPase which is a membrane-bound (MBE) enzyme that can maintain signals activities and maintain $\mathrm{Na}+, \mathrm{K}+$-ATPase for brain activities [9].

Where, $\mathrm{Na}+, \mathrm{K}+$-ATPase (MBE)is adopting hypertension, and $\mathrm{N}, \mathrm{A}, \mathrm{K}$, ATPase functions is stabilizing cells membranes signals transmission activities, and adopt the negative charges signals transmission which adopt stabilizing brain activities. Stimulation of Na+, K+-ATPase activity (which basically depending on the S6K1 biosynthesis) increases sodium transport across the renal proximal tubule epithelia [10].

What is the relation of S6K1 to high blood glucose (hyperglycemia) or low blood glucose (hypoglycemia) and diabetic coma? Diabetic disease include either hyperglycemia or hypoglycemia but we should not describe diabetes according to hyperglycemic or hypoglycemic but those phenomena reflect the Consumption, cracking and loss of ATPase in biological processes without normal repairs or with decreasing repairs that can reflect deficiency in pyrimidine nucleotides in S6K1 peptides which is the main of ATPase synthesis, where the deficiency in Ser amino acids during FOX activities will lead to decreasing in the PS/TThymine Kinases and in PS/T Cytosine kinases that will lead to Androgen production instead of Estrogen productions that will reflect mutation in S6K1 peptides due to deficiency in pyrimidines and necessary hydrophobic amino acids in their chains, where S6K1 will be directed to inner cells for producing ATPase (mutated ATPase). As mutated ATPase started for performing its activities will reflect increasing in Androgen with decreasing in Estrogen, that will lead to increasing in blood glucose (due to increasing in TCA with decreasing in pyrimidine synthesis) which described as 
hyperglycemia. But hypoglycemia is the phenomena that describe increasing in mitochondrial oxidative processes with decreasing in proper ATPase biosynthesis, that reflect there are enough early ATPase for performing the catalyzing of sugar molecules but without ATPase repairs that later will be turned to hyperglycemia.

Diabetes is the result of a deficiency in Ser amino acids in the Ser/Thr signaling pathway that reflect deficiency in the formation of the kinase PS/TTK and PS/TCK (mTORC1), and reflect a decreasing in the formation of pyrimidine nucleotides (which included in Ser amino acids), which leads to the decreasing in the production of Estrogen either increasing in Androgen synthesis where androgen described as rich of purines (Adenosine and Guanosine) either decreasing in Thymine and Cytosine nucleotides . So S6K biosynthesis with deficiency in Ser amino acids will produce a mutated S6K1 peptides with rich of purines and deficiency of pyrimidines that will produce mutated ATPase without normal repair.

Antibodies are immunoglobulins proteins which can be classified into ribosomal and non-ribosomal peptides and differentiate according to their amino acids Containments (quality and quantity). Each of those antibody peptides when oxidized by OPA1 enzymes will generate the three fatty-acyl-CoA isoforms (gamma, beta and alpha subunits), that alpha isoforms contain alpha + beta + gamma, but beta isoforms contain beta and gamma isoforms, while gamma contain only gamma isoforms, where each of those acyl-CoA isoforms are necessary for regulating and activating macrophages and T-cells and consequently necessary for myeloid and lymphoid cells activities for proper SIRP productions, for MHC Class-I synthesis which promote the endocytic soluble MHC Class II synthesis. Those necessary acyl-CoA three isoforms can be functioned directly by OPA1 enzymes for glucocorticoids and for the three interferons isoforms re-synthesis, then for SIRPgamma production which will be for MHC class- 1 which regulate endocytic soluble MHC class II synthesis which will promote the endocytic SIRP $\alpha 1$ synthesis for TLR4 which is necessary for endocytic proliferation roots and activities.

Insulin has two chains A\&B that A and B chains become linked together by two sulfur-sulfur (disulfide) bonds. Pro-insulin, insulin, and $\mathrm{C}$ peptide are stored in granules in the beta cells to be released in capillaries [10], the two A\&B chains are considered as two kinases protein one is "A" formed from Ser and the other "B" is formed from Thr amino acids. Where, "B" chain is poor of pyrimidines Containment but rich of purines (that has the ability to recover S6K1 for recovering ATPase and GTPase), while the other chain " $\mathrm{A}$ " is rich of pyrimidines (Thymine and Cytosine) where, both subunits' chains are bonded with sulfur=sulfur bonds within betacells for beta-cells functions and secretion.

Immune consists of two types of lymphocytes: T and B cells, that $\mathrm{T}$ cells can produce a large number of cytokines through feedback upon the activity of MHC class-I which include and involve synthetase activities, where B cells require specific differentiations and activation conditions to produce cytokines [11]. The beta cells can only produce only kinds of cytokines: 1) PS/T-Thymine Kinase and PS/T-Cytosine kinase from "A" chain, while B chain produce 2) PS/T-Adenosine kinases and PS/T-Guanosine kinases which can switch the production of antibody isotypes in B cells, differentiation of helper T cells which regulated by cytokines [12].

B chain has the function of recover S6Ks peptides production depending on the availabilities of amino acids composition.
Antibodies peptides can be classified to three types of antibodies depending on their Compositions from amino acids and fatty acids:

- $\quad$ Type AB-gamma

- $\quad$ Type AB-beta

- $\quad$ Type AB-alpha

First AB gamma which include synthetase enzyme that can analyze and destroy beta cells (in the absence of "A" chain) and hepatic cells, but AB-beta activate Beta-cells and IFN-beta and can protect hepatic and beta-cells and can increase anti-inflammations processes (through beta-oxidations), but $\mathrm{AB}$-alpha can be activated upon phospholipase effects on AB-beta for accelerating proliferation through producing the endocytic SIRP-alpha for promoting the endocytic TLR4 synthesis that regulate plasma membrane synthesis and endocytic proliferations processes.

When, pyrimidines decreased in nutrition in vivo, the $\mathrm{AB}$-alpha will be enhanced by both synthase and synthetase specifically by synthetase for reactivating gamma-oxidations for reactivating pyrimidine synthesis for hydrophobic amino acids synthesis for promoting and recover the "A "chain synthesis Which can regulate "B" chains in insulin in beta-cells that regulated by synthase effects for Beta-subunit synthesis for AB-beta synthesis which can activate B-cells for promote the GC-beta productions, and for IFN-beta productions. Then IFN-beta will regulate MHC-class-I productions and then the endocytic MHC class II synthesis which will promote the endocytic SIRP $\alpha 1$ synthesis upon the phospholipase effects, SIRP $\alpha 1$ will promote the TLR4 synthesis respectively. That the alpha-oxidations are the necessary processes needed for proliferation including SIRP $\alpha 1$ productions which necessary for TLR4 synthesis and regulations. B lymphocytes (B-cells) can activate a type of white blood cell through the of stimulations of beta-oxidations by OPA1 synthase enzymes (that its molecular compositions rich of pyrimidines in hydrophobic acids). It has been reported that preventing hepatic insulin resistance via the adenosine monophosphate-activated protein kinase-p70 ribosomal S6 kinase-1 pathway [13].

That the preventing insulin resistance will need to re-activate protein p70-Kinases for renew the S6K1 molecular composition which will recover the beta-chain in insulin for preventing insulin resistance. S6K1 is necessary for insulin growth that as I mentioned before two types of protein kinases responsible for S6 productivity through Ser /Thr- nutrient-mTOR-FOX signaling pathway which are PS/T-Adenosine kinase and PS/T-Guanosine kinases which are mainly produced from Thr amino acids phosphorylation which are rich of purines for S6K1 synthesis then for both ATPase and GTPase. Hence, S6K1 can activate insulin growth and vice versa. That, the insulin-induced activation of p70 S6 kinase and MAP kinases [14]. Where, amino acids are necessary for the insulin-induced activation of mTOR/S6K1 signaling and protein synthesis to prevent insulin resistant in human skeletal muscle [15].

The insulin resistance or drugs resistance occur due to stability of specific fragments due to increasing in positive linkages that stabilize linkages against antibiotic can resist OPA1 oxidative processes which have to be occurred for renew those +ve fragments for continuing adopting the necessary molecular structure for proper endocytic processes. The resistance to antibiotics can be due to deficiency in Ser amino acids and in pyrimidine nucleotides with decreasing oxidative processes by mitochondrial enzymes, but some cases can be due to reductions in ATPase with reduction in 
S6K1 productions due to decreasing in ser/Thr signaling pathway.

The Containments of specific advantages amino acids as Proline in gamma, beta, and alpha subunits can accelerate proper ATPase activities and OPA1 oxidations which facilitate ifns isoforms productions for MHC-class-I synthesis which regulate the endocytic MHC class II synthesis for SIRP $\alpha 1$ production which necessary for TLR4 synthesis and TGF-gamma/beta/\& alpha synthesis respectively. Where the presence of proline in IFN-gamma, in GC-gamma, in TLR4 genes and in IFN-beta will accelerate their activities and oxidative OPA1 anabolic processes and accelerate the alpha oxidations for SIRP $\alpha 1$ and TLR4 production productions which promote plasma-membranes, collagen synthesis and blood platelets, [16] CD8 T cells as well as other cells can and do produce IFN- $\gamma$ during M. tuberculosis infection [17].

S6K1 consider as a mediator subunit for Interferons (IFNs) isoforms synthesis regulated by pyrimidine-kinases. It has been reported that interferons are a family of cytokine mediators critically involved in alerting the cellular immune system to viral infection of host cells [18]. Interferons are not alerting central immune to viral infections, but interferons are produced for modulating the anti-inflammatory processes (not alerting original basic genes and subunits) which in brief through modulating IFNs synthesis and glucocorticoids biosynthesis, and for Interferon's isoforms synthesis. Ribosomal peptides S6 kinase beta-1 (S6K1) is a result from Serine/Threonine phosphorylation pathway, that has imp roles in immune regulating or promoting the lymphocyte activations, where S6K1/S6 axis participated in the primary response of anti-bacterial adaptive immunity in Nile tilapia [19]. And Interferons-Dependent Engagement of Eukaryotic Initiation Factor 4B via S6 Kinase (S6K)- and Ribosomal Protein S6KMediated Signals [20].

The mRNAs translation for IFN-sensitive clearly are the S6K1 peptides that activate ATPase phosphorylation which back phosphorylate the eIF4B on Ser amino acids for adopting IFN gamma, beta, and alpha compositions through translations according to the basic S6K1 and then related to ATPase sequences composition. Any mutations will occur in S6K1 peptides due to absence of Ser, Arg, proline, Thr, tyr or leu or due to absence or decreasing in pyrimidines can reflect pathogenic symptoms (Figure 4).

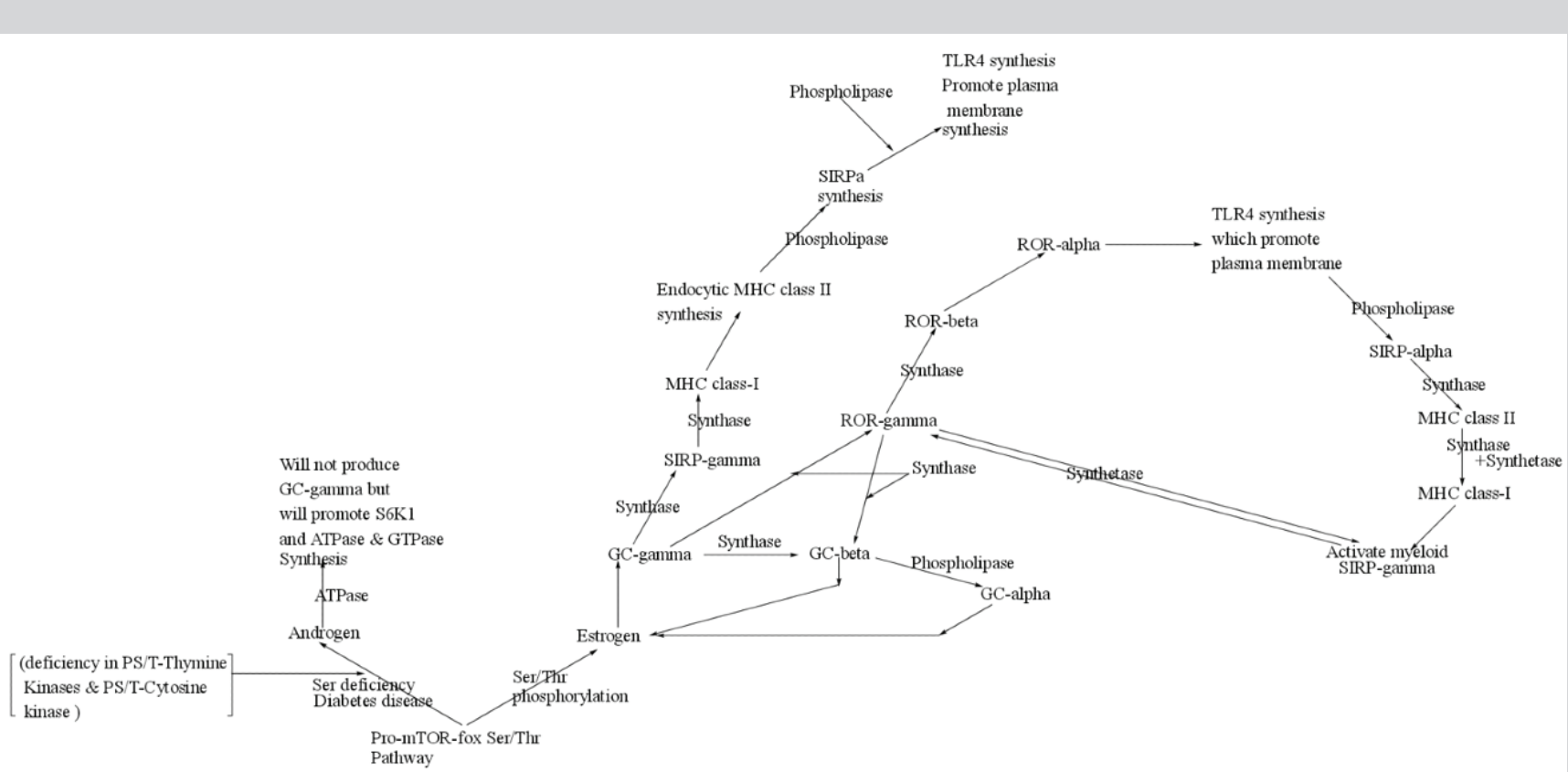

Figure 4: Roles of Estrogen for glucocorticoids synthesis, While Androgen's cannot promote GCs synthesis but through feedback can promote S6K 1 synthesis.

Asthma is characterized by Prediabetes and diabetes, that deficiency in pyrimidines (deficiency in Ser) is the main reason for two diseases: It has been reported that (S6K1) inhibition with rapamycin inhibited IFN- and EGF-induced protein synthesis [21]. In severe Asthma which characterized by increased airway smooth muscle (ASM) that begin due to decreasing in Ser amino acids in pro-nutrients-mTOR-FOX pathways that will reflect decreasing in the PS/T-Cytosine-Kinases (mTORC1) and in PS/T-ThymineKinases production (which are necessary for migrating and regulating molecules too) with increasing in P Ser/Thr-Adenosinekinases with Ser/Thr-Guanosine-kinases (which specified for S6K1 peptides synthesis) that lead to decreasing in migrating molecules due to decreasing in cytosine(decreasing in pyrimidines), and decreasing in tRNAs with increasing in ATPase and GTPase (which repaired and reactivated by S6K1 peptides) that will lead to promote glucocorticoids (from androgen) and interferons synthesis, where their biosynthesis characterized by deficiency in Ser amino acids and in its related kinases (PS/T-Cytosine Kinases and PS/T-Thymne Kinases) productions, that will lead to decreasing in sugar, lipid, LPS, and carbohydrate metabolism (lead to increasing of purines molecules and LPS in blood and interstitium fluid) and conjugated with increasing in S6K1 peptides productions (for ATPase repair) and for growth factor synthesis, that will lead to increasing in airway smooth muscle (ASM), note, patients that having Asthma are having the so high risk of sever diabetic problems due to deficiency of Ser amino acids(deficiency in Ser/Thr phosphorylation pathways) that will lead to deficiency in Estrogen productions with increasing in Androgen productions which are characterized by high purines with deficiency in pyrimidines nucleotides (deficiency in pyrimidine-kinases lead to deficiency in PS/T-Cytosine Kinase and PS/T-Thymine kinases synthesis). 
Consequently, deficiency in pyrimidine can lead to mutations in ribosomal enzymes and mutations in the productions of long fatty-acyl-CoA-isoforms upon OPA1 enzymes functions. So, Asthma is characterized by diabetes symptoms, where, in the early days of Asthma diabetic symptoms cannot be detected but later will be detected. Where, there are limited data on the risk of pulmonary disease in patients with diabetes [22]. And it is concluded that asthma and T2DM are the two common chronic conditions of increasing prevalence and that often coexist in the same patient [23]. Also, it has been reported that: Prediabetes and diabetes have been recently identified as risk factors for asthma exacerbations in adults [24].

Note, in some cases decreasing in Cytosine-kinases and or fattyacyl-CoA-synthetase (gamma-subunits) will reflect decreasing in ATPase repairs (due to cytosine necessary with purines for rebuilding ATPase which is necessary for migrating molecules)that lead to accumulations of subunits within cells and in the interstitium fluid that lead to decreasing in their migrations with increasing in their +ve linkages that lead to tumor growth and coagulation in blood vessels and can reflect other diseases symptoms example: early diabetic symptoms. Where, Advances along the small GTPase front have implicated cell migration [25].

Proper GTPase productions implicate cell migrations and the mitochondrial OPA1 membranes repairs in case of availabilities of both PS/T-Cytosine kinases and PS/T-Thymine Kinases (and in availability of proline and hydrophobic amino acids that accelerate OPA1 anabolic oxidative processes properly) that can increase Tyrosine metabolic pathways and will increase signals transmissions that will promote the migrations of active of genes and subunits which can activate beta-oxidative processes and alpha-oxidations (that regulated by synthase and by phospholipase respectively), that can promote phosphatidic acid (PA) to rebinds to mTOR and to re-binds to S6K peptides independently of mTOR, where S6K peptides synthesis are basically depending on the two purines kinases that produced from Ser/Thr-FOX signaling pathways for producing PS/T-Adenosine $\mathrm{k}$ and PS/T Guanosine kinases for S6K1 synthesis, for ATPase and for GTPase reactivities, and for promoting endocytosis MHC class II functions, where its migration need the availabilities of Cytosine-kinases and Thyminekinases (active pyrimidine-kinases) production for S6K1 peptides productions and for tRNAs synthesis. MHC Class-I is fatty-acyl-CoA gamma-subunits (IFN- gamma) for modifying their fatty acyl-CoAbeta (IFN-beta) synthesis to be site and lie on nucleated cells as MHC class-1.

The IFN-gamma biosynthesis regulated by the effects of synthetase enzymes on lipid and LPS for producing long-fatty acids chains (through gamma-oxidation) for producing fatty-acylCoAsynthetase subunits (IFN-gamma) that promote IFN-beta, where both IFN-gamma and beta have the functions of activating the soluble endocytic MHC class II synthesis which originally produced from MHC class-I and has anti-inflammatory activities that will migrate to nucleated cells to site and lie on their membrane for producing endocytic MHC class II. The biosynthesis of long fattyacyl-CoA-synthetase (ACS) and other beta and alpha isoforms are acting as homodimeric enzymes, that acyl-CoA synthetase member 6, ACSL6, is a form present in the plasma membrane of cells [26].

Where, "ACS" productions are necessary for regulating TLR4 which necessary for plasma membrane synthesis, and necessary for endocytic MHC class II synthesis which originally regulated by fatty-acylCoA-gamma (synthetase functions) and by IFN-gamma that carry the anti-inflammatory function that bind to inflammation contents for modifying their own gamma-subunits for regulating MHC-class-I which followed by the endocytic MHC class II synthesis that is so necessary for endocytic SIRP $\alpha 1$ productions.

IFN-gamma has the functions of anti-inflammatory processes that can analyze inflammation and infected cells for binding to resulted peptides and recreate pyrimidine nucleotides for modifying its own active gamma-subunits and then for regulating IFN-beta synthesis upon the effects of OPA1-synthase enzyme followed by the regulation of MHC-class-I which regulate the endocytic MHC class II synthesis followed by SIRP $\alpha 1$ and TLR4 synthesis respectively.

That MHC class II can be described as the main regulator for reactivating the endocytic TLR4 upon phospholipase. Furthermore, the co-expression of MHC class II molecules and TLR2 or TLR4 in human embryonic kidney (HEK) cells 293 leads to enhanced production of the anti-microbial peptide human- $\beta$-defensin [27]. Previous study indicating the imp fact that MHC class II enhance the endocytic TLR4 reactivities upon phospholipase oxidative effects. Where, MHC-II molecules are important for initiation the antigenspecific immune response where need to be promoted by activating TLR4 for endocytic proliferation then for external proliferations.

Dendritic cells (DCs) initiate adaptive immune responses by activating $\mathrm{T}$ cells via cognate interactions between MHCpeptide complexes and $\mathrm{T}$ cell receptors [28]. where, class II is predominantly stored in endocytic plasma membrane, where it has a short half-life because of its regulation to TLR4 synthesis for Plasma membrane modification and re-synthesis, that is why the plasma membrane which promoted by MHC Class II and formed by TLR4 its Containment is MHC class II, and it has been considered that $\mathrm{T}$ cell growth-promoting activity of interferon-gamma [29]. And also, IFN- $\gamma$ mediates CD8 T-cell cytotoxic function. that in vivo, antigen-specific CD8 $\mathrm{T}$ cells that produce INF- $\gamma$ necessary to effect rejection of skin grafts [30], where, cytokine productions upon FOX activities will be modified by the effects of mitochondrial enzymes to produce specific modified IFN-gamma which has the antiinflammatory effect and binding to selected of result peptides that will reactivate IFN-beta upon synthase oxidations and then will site on and cover nucleoid cells for MHC class II synthesis which will has the role of reactivating TLR4 upon regulation of phospholipase for Plasma membrane synthesis and for TLR4 other functions.

Also, The ability of CD8 T cells to re-produce IFN $-\gamma$ indicating that IFN- $\gamma$ connected to the MHC class II re-activities, and in fact as mentioned above that IFN- $\gamma$ basically has the function of regulating the MHC class II synthesis that during inflammation and infection the IFN- $\gamma$ will analyze inflammations content through gamma-oxidation then will bind to selected resulted peptides for modifying its own subunits then will sit and lie on cell membrane as MHC class-I for reactivating the endocytic soluble MHC class II synthesis, also would like to mention that MHC class II is a fattyacyl-CoA-synthase (Beta-subunit) which regulate and activate TLR4 synthesis upon phospholipase oxidations for producing fatty-acylCoA-phospholipase (alpha-subunits which considered as IFN-alpha necessary for proliferation cycles), that the interferons (IFNs) have functions and diverse effects upon OPA1 oxidations on initiate and adapting immune cells during infection including proliferations function through regulating TLR4 for running proliferations.

In conclusion the previous studies indicated that MHC class-I considered as fatty-acyl-CoA-synthetase (gamma-subunits) and 
considered as IFN-gamma that can analyze infected cells and inflammations then bind to their peptides for modify and promote IFN-beta synthesis upon synthase oxidations covering nucleated cells membranes for reproducing MHC class II which considered as fatty-acyl-CoA-beta (IFN-beta) that upon alpha-oxidations will promote fatty-acyl-CoA-phospholipase which considered IFN-alpha for regulating TLR4 synthesis upon alpha-oxidations for plasma membrane synthesis which contain MHC class II , and then again act on inflammations and on infected cells through feedback for reproducing IFN-beta then IFN-gamma regulated by OPA1 oxidations across cells membrane for acting on inflammation and bind again to their peptides for re-improving their gamma-subunits and Betasubunits (which conclude gamma-subunits in their chains) which can re-modified again and act as MHC class-I which will re-sit and lie on cells membranes for running the soluble endocytic MHC class II synthesis and then TLR4 synthesis.

Where, Thr, Gly, Glu, Arg, Lysine (AAG, AAA), and proline are the most important amino acids peptides for rebuilding necessary fatty-acyl-CoAs isoforms (IFN-isoforms) and for necessary MHC class II that can be stored in lysosomes, for running OPA1 oxidative processes and for TLR4 synthesis to perform proliferations, for adopting neurons response, and for promoting brain cells functions, where MHC class II is regulated by MHC class-I synthesis.

On the other hand, the Tyr, Met, Arg, Ser, Pro, and Leu amino acids are so necessary amino acids for rebuilding the proper MHC class-1 which regulate and promote MHC class II synthesis and then regulate both SIRP $\alpha 1$ and TLR4 biosynthesis for endocytic plasma membrane synthesis and endocytic proliferations.

Signal regulatory protein $\alpha,(\operatorname{SIRP} \alpha)$ is necessary for IFN-alpha synthesis which activate TLR4 for Plasma membrane synthesis where through feedback will produce firstly MHC class II (upon beta-oxidation) followed by gamma-oxidation for producing MHC Class-I. Signal regulatory protein-alpha (SIRP $\alpha 1$ ), also known as Src homology 2 domain-containing phosphatase substrate-1, is expressed on myeloid and hematopoietic stem cells and neurons. Where the production of SIRP $\alpha$ and their activities can increase signals transmissions that can increase migrations and signal transduction, and promote OPA1 alpha oxidations, for cell proliferation, and transformation.

SIRP $\alpha$ has the function of producing IFN-alpha and TLR4 that through feedback will produce firstly endocytic MHC class II and IFN-beta (SIRP-Beta) upon synthase beta-oxidation, then followed by MHC Class-I and IFN-gamma productions (also considered as SIRP-gamma) upon synthetase oxidations that will sit and lie on nucleated cells membranes recreating the MHC class II again and for running the anti-inflammatory functions and internal endocytic activities. Signal regulatory protein $\alpha$ negatively regulates mast-cell activation [31]. And Ligation of SIRP $\alpha$ (on macrophages) by CD47 (on RBCs) promotes tyrosine phosphorylation [32].

SIRP $\alpha$ is negatively regulate mast cells directly but can activate mast cells indirectly through SIRP-gamma production that can reactivate both IFN-gamma and MHC-class-I synthesis (by stem cells), furthermore the SIRP that can promote Tyrosine phosphorylation due to the gamma-oxidation upon synthetase enzyme that activate SIRP-gamma from both beta and alpha subunits that can promote Tyrosine phosphorylation pathways which promote pyrimidine and hydrophobic acids synthesis (Tyr, Leu... etc.). The IFN- $\gamma$-induced surface expression of CD47 contributed to a stronger binding affinity to SIRP $\alpha$ [33]. Where,
IFN- $\gamma$ have the function of promoting MHC-class-I which directed to the nucleated cell membrane for promoting endocytic MHC class II, while has the function of promoting the SIRP-gamma which promote the MHC-class-I too. And this study indicates that the two subsets of CD14+SIRP $\alpha$ had limited ability to migrate and phagocytose; but stimulated T-cell function [34], where, SIRP $\alpha$ has the activities of promoting TLR4 synthesis and proliferation but not migration, while SIRP-gamma has the activation for increasing signals transmission and migrations.

2'-5'oligoadenylate synthases (OASs), a family of latent 2'-5'-adenylyl transferases, otherwise involved in cellular antiviral responses, are also involved in PAR remodeling of the DDR in MDS and AML cells [35]. Myeloid cells are major players that exploit the regulators of Arginine metabolism (for proline synthesis) to mediate diverse and adopt immunity [36]. That fatty-acylCoA-Synthetase in myeloid is so important for adopting TCA cycle (Arg metabolism) which has the function of purines synthesis and proline synthesis, while also synthetase regulate pyrimidine nucleotides synthesis for acyl-CoA synthetase productions and for IFN-gamma productions.

In conclusion I would like to declare imp fact that SIRP $\alpha$ is produced from the endocytic MHC class II and from endocytic IFN-beta which can promote TLR4 synthesis, while SIRP-gamma is secreted from IFN-gamma in myeloid and hematopoietic stem cells and neurons that promote MHC-class-I synthesis and promote the endocytic SIRP $\alpha 1$ production, and has not the ability to activate mast cells that is secreted as so active SIRP Gamma sensor "fattyacyl-CoA-synthetase" that modified by Arg metabolism.

SIRP-gamma upon synthase effects will promote SIRP-2-Beta upon regulations effects of synthase, where both SIRP-gamma and beta will promote the MHC-class-I production which migrated to nucleated cells membranes to sit and lie on for promoting the endocytic soluble MHC class II which upon alpha-oxidations by will produce SIRP $\alpha$ (IFN-alpha) Which will be directly promote TLR4 synthesis for modifying plasma membrane and for necessary endocytic proliferations. The metabolic purpose of fatty acid oxidation is to generate NADH for ATP generation by the regulations of mitochondrial OPA1 membrane oxidative phosphorylation effect [37]. And FFAs are activated via esterification to CoA, which generates a fatty acyl-CoA moiety [38]. Fatty acid oxidation produces acetyl-CoA units which move in this pathway as fatty acylCoA derivatives for utilizing NAD and FAD [39]. So, When OPA1 oxidations is absent it will prevent the fatty-acyl-CoA synthesis and then will increase fatty acids accumulations due to decreasing in the OPA1 oxidations and will decrease or prevent the production of NAD and FAD and consequently reduce Vitamin K metabolic cycle and ATP productivity, that decreasing on ATPase will decrease the lipid, carbohydrate, and protein metabolism through decreasing in FOX pathways and then decreasing in ROR-gamma then decreasing in ROR-beta then decreasing in ROR-alpha which lead to decreasing in proliferation and deficiency in immune functions.

NADH and FADH2 and then the TCA cycle are activated by the electron transport signal transduction which yield from gammaoxidation processes, where NADH and FADH2 produced by both $\beta$-oxidation and the TCA cycle are using the electron transport chain to produce ATP [40].

where, NAD+ deficiency has been found in models of a number of diseases such as cerebral ischemia, myocardial ischemia, and diabetes, and in models of aging NAD+ deficiency is a common central pathological factor in a number of diseases [41]. The 
decreasing or deficiency in S6K1 production will reflect the decreasing in the ATPase which also reflect the decreasing in mitochondrial OPA1-oxidations and decreasing in NAD and FAD that reflect diseases symptoms as Asthma, diabetes, cancers, etc. where NAD+ treatment has been shown to reduce PARP1-induced astrocyte and prevent PARP1-mediated NAD+ depletion in cardiac myocytes in the presence of $\mathrm{H} 2 \mathrm{O} 2$ [42].

NAD is so necessary for MHC class-I synthesis where, the degradation of most cellular proteins occurs by the ubiquitinproteasome pathways peptides generated by the ubiquitinproteasome pathway which are presented by MHC class I molecules [43]. So, NAD and proteasomal pathway has their strong effects on most of proteins degradations and their synthesis which can be necessary for rebuilding MHC class-I subunits which promote endocytic MHC class-II productions.

Estrogen formed from Ser/Thr mTOR Fox signaling pathways for glucocorticoids synthesis. Upon OPA1 enzymes effects on estrogen, but the feedback of GC to produce estrogen will upon ATPase \& cox on GCs isoforms: Fatty acyl-CoA synthetase) that can be used for re-activating glucocorticoid-gamma productions, while the effects of synthase on acyl-CoA-synthetase (gamma subunits) will produce glucocorticoid-beta which can produce IL-beta that reactivate astrocyte, netrin-1, and neutrophiles functions. The glucocorticoid Receptor (GRs) has roles of function of re-activating steroid hormone upon ATPase and cox via ser Thr Fox signaling pathways, and mitochondrial matrix repair and activations, throughout reactivate S6K1 synthesis for ribosomal ATPase and GTPase productions which necessary for OPA1 repair, where glucocorticoid (GC) contains the estrogen receptors that GR has strong roles for estrogen biosynthesis and vise-versa in different pathways the first through ATPase Effects via Ser/Thr FOX pathways for re-producing estrogen, but second through OPA1 oxidative processes on estrogen, and strong roles in antiinflammatory pathways.

The glucocorticoids isoforms (GCs) Biosynthesis pathways is linked to Estrogen biosynthesis and protein kinases which promote Estrogen synthesis. The effects of synthetase on estrogen is for analyzing Estrogen molecules for producing glucocorticoidsgamma subunits and utilizing active signals for re-activating glucocorticoids-beta synthesis upon synthase effects on GCgamma. GC Gamma is the basic units for netrin-1 synthesis which is the basic for the neutrophile repairs and reactivations.

Glucocorticoid's syntheses begin from estrogen upon effects of synthetase for GC gamma production, followed by beta-oxidation by the effects of synthase for producing glucocorticoids-beta and other Beta-subunits that can show enhancing and increasing in the uptake of other beta a myeloid protein [44] followed by phospholipase effects in case of proliferations processes through producing GC alpha.

Glucocorticoid-gamma has anti-inflammatory activities on inflammations for producing pro-inflammatory subunits due to the effects of acyl-CoA synthetase (gamma subunits) on inflammations for analyzing their contents for starting anti-inflammatory pathways activity, and also GC-beta has the same anti-inflammation effect and has the function of SIRP synthesis from myeloid for the MHC class-I production which directed to nucleated cells to lie on and produce the endocytic MHC class II for the SIRP $\alpha$ production and for TLR4 synthesis. GC- beta productions regulate GC-alpha which regulate membrane receptors through activating TLR4 started by SIRP $\alpha$ production. Brocken Estrogen by synthetase can activate a myeloid Beta-subunit synthesis.

In case of diabetic disease is androgen can produce glucocorticoids and vise-versa? In case of androgen synthesis due to deficiency in Ser amino acids lead deficiency in Ser /ThrFox signaling pathway will not produce GC gamma or GC beta upon ATPase Effects (Depending on the \% of Ser deficiency) but will produce GC alpha that also can produce SIRP $\alpha 1$ and TLR4 either a deficiency in their pyrimidine nucleotides but will not produce proper MHC class-I and also the MHC class II will not be produced in nucleated cells that diabetic patients will have a deficiency in T-cells re-activations and in macrophages reactivation. Also, Androgen in diabetes disease can increase mainly the S6K1 resynthesis and ATPase productions and reactivities due to the Androgen is rich of purines and at the same time can increase not proper SIRP $\alpha$ and TLR4 for only reactivating ribosomes and OPA1 repairs with re-increasing in S6 synthesis. Estrogen availabilities can reactivate OPA1 synthetase activities that can promote pyrimidines production for hydrophobic amino acids synthesis, while Androgen cannot promote and cannot activities synthetase functions that can't activate hydrophobic acids synthesis, also has not the abilities to produce both proper GC gamma, beta and SIRP, consequently androgen cannot activate MHC class-I productions and Suppress IFN gamma productions [45].

\section{DISCUSSION AND CONCLUSION}

S6K1 consider as protected basic subunits for ribosomal ATPase, and for Interferons (IFNs) isoforms synthesis regulated by pyrimidine-kinases. The Proper GTPase implicate cell migrations regulated by PS/T-Cytosine kinases and PS/T-thymine Kinases (in availability of proline and hydrophobic amino acids for promotingOPA1 anabolic oxidative processes properly) that can increase Tyrosine metabolic pathways and increase signals transmissions that can promote the migrations of active of genes and subunits which activate beta-oxidative processes and alphaoxidations (that regulated by synthase and by phospholipase respectively), that can promote phosphatidic acid (PA) to re-binds to mTOR and to re-binds to S6K peptides independently of mTORFOX pathways .

Basically, MHC class-I considered as fatty-acyl-CoA-synthetase (gamma-subunits) that regulated by IFN-gamma that promote and regulate SIRP-gamma productions and promote IFN-beta synthesis upon synthase oxidations. MHC-class-I can migrate to nucleated cells membranes to promote endocytic MHC class II that upon alpha-oxidations will promote IFN-alpha and SIRP $\alpha 1$ synthesis for promoting TLR4 synthesis upon the alpha-oxidations for plasma membrane synthesis and for endocytic proliferation. IFN-gamma regulate both MHC-class-I and SIRP-Gamma sensor "fatty-acyl-CoAsynthetase" that modified by Arg metabolism that stimulate and promote anti-inflammatory processes and regulate the productions of SIRP-2-Beta (IFN-beta) upon regulations of synthase which activate MHC-class-I production to be directed to nucleated cells membranes to sit and lie on for producing MHC class II that will promoted.

SIRP-Beta and SIRP $\alpha$ (IFN-alpha) which promote TLR4 synthesis for modifying plasma membrane and for necessary proliferations when upon feedback will reproduce the MHC class-I. The decreasing or deficiency in S6K1 productions will reflect the decreasing in the ATPase (can be detracted after consuming the stored adenosine nucleotides in producing ATPase) and reflect decreasing in fatty 
acyl-CoAs production which regulated by OPA1 oxidative function that can reflect decreasing in NAD and FAD which can start the Asthma, diabetes, cancers diseases. The effects of synthetase on Estrogen will activate glucocorticoids isoforms synthesis start by GC-gamma productions followed by glucocorticoids-beta upon synthase effects on GC-gamma. GC Gamma is the basic units for netrin-1 synthesis which is the basic for the neutrophile repairs and reactivations. SIRP-gamma regulated by IFN-gamma where both regulate MHC class-I synthesis which will be migrated to nucleated cells for MHC class II that will regulate SIRP-beta synthesis which upon alpha-oxidations by phospholipase effects will produce SIRP $\alpha$ that will be promote TLR4 synthesis.

Estrogen is the substrate for glucocorticoids biosynthesis that GCs isoforms can recover IFNs isoforms and vice-versa. Myeloid cells are major players that exploit the regulators of Arginine metabolism (for proline synthesis) to mediate diverse and adopt immunity. That fatty-acylCoA-Synthetase in myeloid is so important for adopting TCA cycle (Arg metabolism) which has the function of purines synthesis and proline synthesis, and synthetase control the pyrimidine synthesis for hydrophobic amino acids synthesis Tyr, Leu, Ser, Arg, Pro, etc.

\section{CONFLICT OF INTEREST STATEMENT}

The Author declare that the research work has been conducted in the absence of any commercial or financial relationships, that could be construed as a potential conflict of interest.

\section{REFERENCES}

1. Zhang X, Rubin J, Henry L, Sen X (2020) Feeding tricarboxylic acid cycle intermediates improves lactate consumption and antibody production in Chinese hamster ovary cell cultures. Biotechnol Program 36(4): e2975.

2. Tantawi AME (2021) OPA1 is basis for macrophages, glucocorticoids subunits, neutrophile, RAW264.7 Cells and T-Cells regulations, where OPA1 contain COX sequence followed by synthetase, then synthase, then phospholipase sequences respectively. International Journal on Pharmacy and Sciences 1(2): 52-61.

3. Jordan TN, Mitchell RA (2019) Tricarboxylic acid cycle metabolites in the control of macrophage activation and effector phenotypes. Journal of leukocyte Biology 106(2): 359-367.

4. Williams NC, O’Neill LAJ (2018) A role for the krebs cycle intermediate citrate in metabolic reprogramming in innate immunity and inflammation. Frontiers in Immunology 9: 141.

5. Aleksandra HAC, Karolina P, Anna HA (2021) S6K1 is indispensable for stress-induced microtubule acetylation and autophagic flux. Cells 10(4): 929.

6. Hassan AA, Ding Y, Bixby JL, Tatiana S, Wei W, et al. (2017) The mTOR substrate $\mathrm{S} 6$ kinase 1 (S6K1) is a negative regulator of axon regeneration and a potential drug target for central nervous system injury. The journal of Neuroscience 37(30): 7079-7095

7. Devi SS (2010) 9.02 - Structure and function of hepatic parenchymal cells. Comprehensive Toxicology 9: 11-30.

8. Aslan JE (2011) S6K1 and mTOR regulate Rac1-driven platelet activation and aggregation. Blood 118(11): 3129-3136.

9. Rezin GT, Streck EL, Giselli S, Cinara LG, Gabriela KF, et al. (2014) Evaluation of $\mathrm{Na}+, \mathrm{K}+$-ATPase activity in the brain of young rats after acute administration of fenproporex. Brazilian Journal of Psychitary 36(2): 138-142.

10. Jaitovich A, Bertorello AM (2010) Salt, $\mathrm{Na}+\mathrm{K}+-\mathrm{ATPase}$ and hypertension. Life Sciences 86(3-4): 73-78.

11. Vazquez MI, Beta J, Zlotnik A (2015) B cells responses and cytokine production are regulated by their immune microenvironment. Cytokine 74(2): 318-326.
12. Tangye SG, Anthea F, Danielle TA, Cindy SM, Philip DH (2002) Isotype switching by human B-cells Is division-associated and regulated by cytokines. The Journal of Immunology 169(8): 4298-4306.

13. Bae EJ, Yoon MY, Jin WK, Sang GK (2007) Identification of a novel class of dithiolethiones that prevent hepatic insulin resistance via the adenosine monophosphate-activated protein kinase-p70 ribosomal S6 kinase-1 pathway. Hepatology 46(3): 730-739.

14. Wijkander J, Landström TR, Manganiello V, Belfrage P, Degerman E (1998) Insulin-induced phosphorylation and activation of phosphodiesterase $3 \mathrm{~B}$ in rat adipocytes: possible role for protein kinase B but not mitogenactivated protein kinase or p70 S6 kinase. Endocrinology 139(1): 219227.

15. Drummond MJ, Jill AB, Satoshi F, Hans CD, Erin LG, et al. (2008) Amino acids are necessary for the insulin-induced activation of mTOR/S6K1 signaling and protein synthesis in healthy and insulin resistant human skeletal muscle. Clinical Nutrition 27(3): 447-456.

16. Ashraf MET (2021) Toll-like receptor-4, synthesis is regulated by JNK signaling, by three glucocorticoids isoforms and by three interferons isoforms, also its advantages in activities depending on the containment from Arg, Proline and hydrophobic amino acids in its compositions. Open access Journal of Biomedical Science 4(4): 1125-1133.

17. Yoon JW (2005) Autoimmune destruction of pancreatic beta cells American Journal of Therapeutics 12(6): 580-591.

18. Hiscott J, Le Page C, Génin P, Baines M G (2000) Interferon activation and innate immunity. Reviews in Immunogenetics 2(3): 374-386.

19. Li K, Xiaotong S, Hong Q Tianyu Z, Kete A, et al. (2020) S6K1/S6 axisregulated lymphocyte activation is important for adaptive immune response of Nile tilapia. Fish and Shellfish Immunology 106: 1120-1130.

20. Barbara K, Surinder K, Efstratios K, Beata MK, Antonella S, et al. (2009) Interferon-dependent engagement of eukaryotic initiation factor $4 \mathrm{~B}$ via S6 kinase (S6K)- and ribosomal protein S6K-mediated signals. Molecular and Cellular Biology 29(10): 2865-2875

21. Goncharova E, Poay NL, Amelia C, Homer WF, Jerome HT, et al. (2010) Interferons modulate mitogen-induced protein synthesis in airway smooth muscle. Lung Cellular and Molecular Physiology 299(1): 25-35.

22. Ehrlich SF, Charles PQ, Stephen KV, Jun S, Assiamira F (2010) Patients diagnosed with diabetes are at increased risk for asthma, chronic obstructive pulmonary disease, pulmonary fibrosis, and pneumonia but not lung cancer. Diabetes Care 33(1): 55-60.

23. Torres R, Marcela Dos SS, Ana Carla CC, Luane MM, Carolina SM (2021) Association between asthma and type 2 diabetes mellitus: Mechanisms and impact on asthma control-A literature review. Canadian Respiratory Journal 2021: 8830439

24. Wu TD (2020) Diabetes and glycemic dysfunction in asthma. The Journal of Allergy and Clinical Immunology: In Practice 8(10): 3416-3147.

25. Julian GC (2010) New concepts in phospholipase D signaling in inflammation and cancer. The Scientific World Journal 10: 1356-1369.

26. Soupene E, Dinh NP, Kuypers FA (2010) Activity of the acyl-CoA synthetase ACSL6 isoforms: Role of the fatty acid Gate-domains. BMC Biochemistry 11: 18.

27. Frei R, Johanna S, Thomas B, Susanne L, Caroline R, et al. (2010) MHC class II molecules enhance toll-like receptor mediated innate immune responses. PLOS One 5(1): e8808.

28. Niel GV, Richard W, Toine Ten B, Sonja IB, Ferry AO, et al. (2006) Dendritic cells regulate exposure of MHC Class II at their plasma membrane by oligoubiquitination. Immunity 25(6): 885-894.

29. Alfinito F, Lamberti A, Capobianchi MR, Nigro M, De Rosa G, et al. (1994) T cell growth-promoting activity of interferon-gamma. Mitogenic effect of the recombinant cytokine on cells from a human T-chronic lymphocytic leukemia. Leukemia 8(8): 1294-1300.

30. Bhat P, Graham L, Nigel W, Ian HF (2017) Interferon- $\gamma$ derived from cytotoxic lymphocytes directly enhances their motility and cytotoxicity. Cell Death and Disease 8: e2836.

31. Pan YF, Li-wei D, Min W, Guang-zhen Y, Jian Z, et al. (2013) Signal 
regulatory protein $\alpha$ negatively regulates mast-cell activation following FceRI aggregation. European Journal of Immunology 43(6): 1598-1607.

32. Murata Y (2014) The CD47-SIRP $\alpha$ signaling system: its physiological roles and therapeutic application. The Journal of Biochemistry 155(6): 335-344.

33. Ye ZH, Xiao-Ming J, Mu-Yang H, Yu-Lian X, Yu CC, et al. (2021) Regulation of CD47 expression by interferon-gamma in cancer cells. Translational Oncology 14(9).

34. Wu H, Ya PC, Hyo JK, Tammy PT, Jose CV, et al. (2019) SIRP $\alpha$ expression delineates subsets of intratumoral monocyte/macrophages with different functional and prognostic impact in follicular lymphoma. Blood Cancer Journal 84: 9.

35. Hasipek M, Yihong G, Dale G, Xiaorong G, Yogenthiran S, et al. (2020) Role of oligoadenylate synthetases in myeloid neoplasia. Blood 136: 29-30.

36. Berg JM, Tymoczko JL, Stryer L (2017) Arginine metabolism in myeloid cells shapes innate and adaptive immunity. Frontiers in Immunology 8: 93.

37. Jagdip S, Wendy K, Wei W, John RU, Gary DL, et al. (2011) Targeting fatty acid and carbohydrate oxidation- A novel therapeutic intervention in the ischemic and failing heart. Biochimica et Biophysica Acta (BBA)Molecular Cell Research 1813(7): 1333-1350.

38. Kumari A (2018) Beta oxidation of fatty acids pages. Sweet Biochemistry, pp. 17-19.
39. Kumari A (2018) Fatty acid biosynthesis. Sweet Biochemistry, pp. 21-24.

40. Fillmore N, Alrob OA, Lopaschuk GD. Fatty acid beta-oxidation. AOCS Lipid Library.

41. Zhang M, Ying W (2018) NAD+ deficiency is a common central pathological factor of a number of diseases and aging: mechanisms and therapeutic implications. Antioxidant and Redox Signaling 30(6): 890905.

42. Massudi H, Ross G, Gilles JG, Nady B (2013) NAD+ metabolism and oxidative stress: The golden nucleotide on a crown of thorns. Redox Report 17(1): 28-46.

43. Leone P, Eui-Cheol S, Federico P, Angelo V, Franco D, et al. (2013) MHC Class I antigen processing and presenting machinery: Organization, function, and defects in tumor cells. JNCI: Journal of the National Cancer Institute 105(16): 1172-1187.

44. Li R, Shen Y, Yang LB, Lue LF, Finch C, et al. (2000) Estrogen enhances uptake of amyloid beta-protein by microglia derived from the human cortex. Journal of Neurochemistry 75(4): 1447-1454.

45. Lin AA, Wojciechowski SE, Hildeman DA (2010) Androgens suppress antigen-specific $\mathrm{T}$ cell responses and IFN $\gamma$ production during intracranial LCMV infection. Journal of Neuroimmunology 226(1-2): 8-19. 